\title{
Tongfu Huoxue Decoction Reduces Autophagy and Apoptosis to Protect Against Acute Renal Injury in a Rat Model of Sepsis
}

\section{Yan Wang}

Hebei University of Chinese Medicine

\section{Zixuan Wang}

North China University of Science and Technology

Qianlan Yang

North China University of Science and Technology

\section{Yuxin Fan}

Hubei University of Chinese Medicine

\section{Lu Wang}

Hebei North University

\section{Yunhe Zhang}

North China University of Science and Technology

Lijian Du ( $\sim$ dulijian7400@163.com )

Shijiazhuang Hospital of Traditional Chinese Medicine

\section{Research}

Keywords: Acute kidney injury in sepsis, Tongfu Huoxue decoction, Autophagy, Apoptosis

Posted Date: December 2nd, 2020

DOl: https://doi.org/10.21203/rs.3.rs-117890/v1

License: (c) (i) This work is licensed under a Creative Commons Attribution 4.0 International License. Read Full License 


\section{Abstract}

Background: To investigate the mechanism by which Tongfu Huoxue decoction protects against acute kidney injury in sepsis in rats, and provide a new therapeutic strategy for clinical intervention.

Methods: Thirty-two Sprague-Dawley rats were randomly divided into a control group (saline), model group (lipopolysaccharide LPS group), LPS group+Tongfu Huoxue Decoction high-dose group (H group), and LPS group +Tongfu Huoxue Decoction low-dose group ( $L$ group). A rat model of acute kidney injury in sepsis was established by administration of LPS, and Tongfu Huoxue decoction was administered by gavage. The pathological and morphological changes of the kidneys were evaluated according to the levels of urea nitrogen (BUN) and blood creatinine (SCr). The expression of the inflammation-related factors IL-1b, IL-6 and TNF-a was determined by quantitative real-time PCR. Changes in the phosphorylation levels of the autophagy and apoptosis-related proteins LC3, beclin-1, caspase-3 and Akt were detected by Western blot analysis.

Results: Compared with the model LPS group, kidney tissue damage was reduced in rats treated with Tongfu Huoxue decoction. The expression levels of inflammation-related and autophagy-related proteins in the kidney tissue of rats treated with Tongfu Huoxue decoction were significantly lower than those in the LPS group, which was showed a dose-dependent decrease in expression. After stimulation of HK-2 cells with LPS, the expression levels of the autophagy and apoptosis in the groups treated with Tongfu Huoxue decoction decreased in a dose-dependent manner. Furthermore, the rate of HK-2 cell apoptosis was higher in the LPS group than that in the control group, with lower rates in the $\mathrm{H}$ and $\mathrm{L}$ groups than that in the LPS group and were dose-dependent.

Conclusion: Tongfu Huoxue decoction protects against acute renal injury in sepsis by reducing autophagy and apoptosis.

\section{Introduction}

Sepsis is a systemic inflammatory response syndrome, which, in the absence of timely intervention, will eventually lead to organ dysfunction. Clinical studies have shown that the kidney is the most severely affected organ in sepsis. Acute kidney injury (AKI) is more common in the early stage of sepsis, with an incidence rate of $55 \%-73 \%{ }^{[1-2]}$, and approximately $70 \%$ of infectious AKI cases are fatal ${ }^{[3]}$. Therefore, protecting the kidney is essential to improve the outcomes of patients with sepsis ${ }^{[4]}$. At present, the mechanism underlying the effect of the traditional Chinese medicine on AKI caused by sepsis is unclear. In this study, we established a rat model of acute renal injury in sepsis to explore the mechanism by which Tongfu Huoxue decoction can be used to treat AKI, and to provide a theoretical reference for clinical intervention in AKI in sepsis.

\section{Materials And Methods}

\subsection{Experimental drugs}


Using the guidelines ${ }^{[5]}$ for equivalent dose conversion between animals and humans in pharmacological tests, the dose of Tongfu Huoxue decoction was calculated as follows: Low dose (rhubarb $9 \mathrm{~g}$, mirabilite $6 \mathrm{~g}$, astragalus $9 \mathrm{~g}$, Salvia miltiorrhiza $9 \mathrm{~g}$, chuanxiong $9 \mathrm{~g}$ ) and High dose (rhubarb $42 \mathrm{~g}$, mirabilite $40 \mathrm{~g}$, astragalus $42 \mathrm{~g}$, Salvia miltiorrhiza $42 \mathrm{~g}$, chuanxiong $42 \mathrm{~g}$ ). For decoction of the experimental drug, the components were placed in a flask and immersed in drinking water to a level of approximately $2 \mathrm{~cm}$ above the dry ingredients, and soaked for $30 \mathrm{~min}$. The mixture was then heated to boiling point before the heat was reduced to a simmer for $30 \mathrm{~min}$. The liquid was then collected and the solid components immersed in water for a second time. The heating procedure was repeated and the two decoctions were pooled before being concentrated under to $200 \mathrm{~mL}$. The doses of crude drug in the high and low dose groups were $2.2 \mathrm{~g} / 1 \mathrm{~mL}$ and $1.3 \mathrm{~g} / 1 \mathrm{~mL}$, respectively.

\subsection{Laboratory animals}

All procedures involving experimental animals were conducted in accordance with the principles of animal experiment ethics and approved by the Animal Experiment Committee of Hebei Medical University (China). Thirty-two specific pathogen-free male Sprague-Dawley rats weighing $240 \pm 10 \mathrm{~g}$ were purchased from experimental Animal Center of Hebei Medical University. The rats were maintained at $23 \pm 2^{\circ} \mathrm{C}$ under a 12-h light/dark cycle with free access to food and drinking water. Experiments were conducted after one week of adaptation to the environment.

\subsection{Experimental cell line and reagents}

HK-2 cell lines were purchased from the American Type Culture Collection (ATCC). PCR kits were purchased from Promega (Madison, Wisconsin, USA) and BCA protein concentration test kits were from Beijing Solarbio Company (Beijing, China). Antibodies for the detection of LC3, Akt, p-Akt, beclin-1, caspase-3, and GAPDH were purchased from Cell Signaling Technology(Danvers, MA,USA). Dulbecco's modified Eagle's medium (DMEM) and fetal bovine serum (FBS) were purchased from Gibco (Grand Island, NY, USA). Lipopolysaccharide (LPS) was purchased from Sigma-Aldrich (St. Louis, MO,USA). The PE Annexin V Apoptosis Detection Kit I was purchased from BD (San Jose, CA,USA). All other biochemical reagents used were domestically produced and analytical grade.

\subsection{Animal grouping and sepsis AKI model preparation}

Thirty-two rats Sprague-Dawley rats were randomly divided into the following groups: control group, LPS group, LPS group + Tongfu Huoxue decoction high-dose group (H group), and LPS group + Tongfu Huoxue decoction low-dose group ( $L$ group), with eight rats in each group. To establish the AKI model, rats in the LPS group were intraperitoneally injected with LPS (dissolved in $0.9 \%$ normal saline, 10 $\mathrm{mg} / \mathrm{kg}$ ). Control rats were injected with the same amount of normal saline. Once the model was established, rats in the high- and low-dose Tongfu Huoxue decoction groups were treated with the corresponding doses for 3 days, while rats in the control and LPS groups were perfused with normal saline at the same time. Subsequently, all animals were allowed free access to food and drinking water. 
Rats were euthanized by intraperitoneal injection with pentobarbital sodium (50 mg/kg). Serum samples and kidneys were collected and stored at $-80^{\circ} \mathrm{C}$ for further analysis.

\subsection{Blood urea nitrogen (BUN) and serum creatinine $(\mathrm{SCr})$ tests}

BUN and SCr levels were measured by an automatic biochemical analyzer (TC6010L, Jiangxi Taikang Technology Co., Ltd., China) using the urease and picric acid methods, respectively. Scr and B. The AKI model was considered to be successfully established when the SCr level in the LPS group increased to twice that in the control group.

\subsection{Observation of the morphological changes in renal tissue}

Renal tissue collected from the rats in each group was fixed with $4 \%$ paraformaldehyde for $24 \mathrm{~h}$, dehydrated using a standard method, and embedded in paraffin wax. Sections ( $4 \mathrm{~mm}$ thickness) were prepared and stained with hematoxylin and eosin $(\mathrm{HE})$. For each section, the pathological changes were evaluated and photographed in five randomly selected visual fields viewed under an optical microscope (OLYMPUS ).

\subsection{Detection of the inflammation-related factors IL-1b, IL-6, and TNF-a}

Total RNA was extracted from the kidney tissue using an extraction kit (Biotek, Winooski, VT, USA) according to the manufacturer's instructions. The mRNA concentration was measured using a ScanDrop 100 (AnalytikJena, Thuringia, Germany). After successful reverse transcription (Table1, Table2), the cDNA generated was used as the template for real-time qPCR analysis using SYBR® Green I (Promega, USA) (Table3). The relative expression of the target gene was calculated using $2^{-\Delta \Delta C t}$ method using $b$-actin as the reference gene. Applied Biosystems company.

\subsection{Western blot analysis of LC3, beclin-1, caspase-3, and p-AKT protein expression}

The kidney tissue collected from each group of rats was homogenized on ice. The protein lysate was placed on ice for $60 \mathrm{~min}$ and then centrifuged for $30 \mathrm{~min}$ at $12,000 \mathrm{r} / \mathrm{min}$ at $4^{\circ} \mathrm{C}$. The protein content in the supernatant was determined using a BCA (50T) kit (Solarbio, Beijing). A protein sample $(30 \mu \mathrm{g})$ was separated by sodium dodecyl sulfate-polyacrylamide (10\%) gel electrophoresis (SDS-PAGE) and transferred ( $90 \mathrm{~V}$ for $1-2 \mathrm{~h}$ ) to a polyvinylidene (PVDF) membrane before blocking in $5 \%$ dried skimmed milk solution at $37^{\circ} \mathrm{C}$ for $2 \mathrm{~h}$. The membrane was then incubated overnight at $4^{\circ} \mathrm{C}$ with the following primary detection antibodies dilute in TTBS buffer: LC3 $(1: 1,000)$ beclin-1 $(1: 1,000)$, caspase-3 $(1: 500)$, pAKT $(1: 1,000)$, p-Akt $(1: 1,000)$ GAPDH $(1: 1,500)$. After washing with TTBS for 5 minutes (3 times), membranes were incubated with secondary detection antibody (peroxidase-labeled, 1:5,000)for 90 min at $37^{\circ} \mathrm{C}$. Membranes were washed three times with TTBS for $10 \mathrm{~min}$ and once for $5 \mathrm{~min}$. The immunoreactive bands were detected and analyzed using the Odyssey imaging system.

\subsection{HK-2 Cell culture}


The cells were inoculated in medium containing double antibody and 10\% FBS high sugar DMEM and placed in $37^{\circ} \mathrm{C}, 5 \% \mathrm{CO}$ medium $2.25 \%$ EDTA was digested and passaged. HK-2 cells were inoculated with $1 \times 10^{5} / \mathrm{L}$ in a 6 -well plate. When cells grew to $70 \% \sim 80 \%$ density, they were cultured with LPS $(10 \mathrm{mg} / \mathrm{L})$ for $12 \mathrm{~h}$; and then with Tongfu Huoxue recipe (high-dose and low-dose groups) for $48 \mathrm{~h}$. The total protein was collected and detected by Western blotting

\subsection{Flow cytometric analysis of apoptosis}

HK-2 cells were seeded into in a 6-well plate $\left(1 \times 10^{5} / \mathrm{ml}\right)$ and treated in the following groups: the control group, LPS stimulation group, high-dose group and low-dose group. The cells were then digested with $0.25 \%$ trypsin, and pipetted to form a single cell suspension before centrifugation at 1,500 rpm for 5 min. The supernatant was discarded and the cells were washed twice with pre-cooled PBS. The cells were resuspended in $1 \times$ binding buffer (Annexin $\mathrm{V}$ binding buffer $10^{\prime}, 50 \mathrm{ml} 0.1 \mathrm{M}$ Hepes/ $\mathrm{NaOH}$ (pH 7.4) $1.4 \mathrm{M}$ $\left.\mathrm{NaCl}, 25 \mathrm{mM} \mathrm{CaCl}_{2}\right)$ at a cell concentration of $1 \times 10^{6} / \mathrm{mL}$. A sample of the single cell suspension $(400 \mu \mathrm{L}$ containing $1 \times 10^{5}$ cells) was then incubated with $1 \mu \mathrm{L}$ PE Annexin $\triangle$ and $5 \mu \mathrm{L}$ 7-AAD with gentle oscillation for $15 \mathrm{~min}$ at room temperature in the dark. The staining reaction was terminated by the addition of 400 $\mu \mathrm{L} 1 \times$ binding buffer and the cells were analyzed within $1 \mathrm{~h}$ on a (Amnis ${ }^{\circledR}$ ImageStream $\left.{ }^{\circledR} \mathrm{X} M \mathrm{MK} \otimes\right)$ (Billerica, MA, USA) flow cytometer using Flow Jo 7.6 software for data analysis. Each experiment was repeated at least three times.

\section{Statistical analysis}

All data were presented as the mean \pm standard deviation (SD) and analyzed using SPSS 17.0. The measurement data were analyzed by $t$-test and $P<0.05$ was set as the threshold for statistical significance.

\section{Results}

\subsection{BUN and SCr levels}

As shown in Figure 1, the levels of serum BUN and SCr in the LPS, LPS $+H$ and LPS $+\mathrm{L}$ groups were higher than those in the control group. The levels of serum BUN and SCr in the LPS $+H$ and LPS $+\mathrm{L}$ group were lower than those in the LPS group, and the levels in the LPS $+H$ group were lower than those in the LPS $+\mathrm{L}$ group. (All $P<0.05$ ).

\subsection{Pathological changes in renal tissue}

As shown in Figure 2, the structure of the renal tissue in the control group was complete and clear, and no obvious inflammatory cell infiltration of the stroma. The cytoplasmic staining of epithelial cells of renal tubules was uniform. The nucleus was in the located centrally within cells, and no obvious degeneration or necrosis were observed. In the LPS group, a large number of renal tubular epithelial cells were 
denatured and necrotic, and there was greater inflammatory cell infiltration of the stroma. Compared with the LPS group, a lesser degree of renal tissue damage was observed in groups $\mathrm{H}$ and $\mathrm{L}$.

\subsection{Tongfu Huoxue decoction reduced the production of proinflammatory factors in sepsis AKI model rats}

Inflammation is one of the most important mechanisms of renal injury in sepsis. To evaluate the ability of Tongfu Huoxue decoction to ameliorate LPS-induced kidney inflammation in rats, we used RT-qPCR to analyze the expression of proinflammatory factors in kidney tissue at the mRNA level. Compared with the control group, significantly higher expression of IL-1 $\beta$, IL- 6 and TNF-a was detected in the kidney tissues of rats in the LPS group, while the expression of these factors was decreased by administration of Tongfu Huoxue decoction (Figure 3). All these results suggested that Tongfu Huoxue decoction alleviates the inflammation associated with AKI in sepsis in rats.

\subsection{Changes in the expression of the autophagy-related proteins LC3, beclin-1, active caspase-3 and p-Akt in rat kidney}

As shown in Figure 4, higher levels of the autophagy-related proteins LC3 $/$ / , beclin 1, caspase 3 and $p$ -

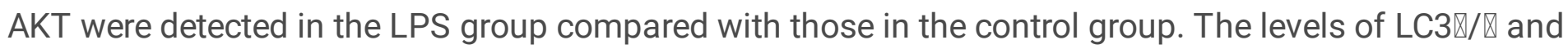
beclin-1 in the LPS+H and LPS+L groups were higher than those in the control group. In contrast, the levels of caspase- 3 and $p-A K T$ in the LPS $+H$ and LPS $+L$ groups were lower than those in the LPS group, with the lowest levels detected in the LPS + H group. (All $P<0.05$ ).

\subsection{Detection of autophagy-related protein LC3 and Akt phosphorylation in vitro}

HK-2 cells were stimulated with LPS $(10 \mathrm{mg} / \mathrm{L})$ for $12 \mathrm{~h}$ before Tongfu Huoxue decoction (high-dose group $\mathrm{G}$ group and low-dose group D) was added to the culture medium. After $48 \mathrm{~h}$, Western blot analysis revealed that the levels of LC3 $/ \triangle$ and $p-A k t$ in the LPS $+G$ and LPS $+D$ groups were lower than those in the LPS group. Furthermore, the levels of LC3\/囚 and p-Akt in the LPS+G group were lower than those in the LPS+D group $(P<0.05)$ (Figure 5).

\subsection{The effect of Tongfu Huoxue decoction on HK-2 cell apoptosis stimulated by LPS}

As shown in Figure 6, flow cytometric analysis showed a higher level of HK-2 cell apoptosis in the LPS group than that in the control group. In contrast, the levels of HK-2 cell apoptosis in the LPS+H and LPS $+L$ groups were lower than those in the LPS group. Furthermore, the level of HK-2 cell apoptosis in the LPS $+\mathrm{H}$ was lower than that in the LPS+L. (All $P<0.05$ ).

\section{Discussion}

Sepsis, which occurs as a result of an aberrant reaction to infection, is the main cause of AKI, accounting for about $50 \%$ of such cases in the in Intensive Care Unit (ICU) ${ }^{[6-7]}$. Sepsis-related AKI is associated with a 6- to 8-fold increase in the risk of death in patients with sepsis, as well as an increase in the progressive incidence of chronic kidney disease (CKD) ${ }^{[8-9]}$. The development and progression of acute kidney injury 
in sepsis is a complex process involving multiple mechanisms. Despite numerous studies exploring new treatment strategies and treatment options, there is still no specific treatment for infectious AKI.

Rhubarb and mirabilite are the main components of Tongfu Huoxue decoction. This formulation is based on the principle of clearing heat and purging turbidity, dispelling blood stasis, creating new, attacking and accumulating stagnation and improving the microcirculation ${ }^{[10]}$. Ligusticum chuanxiong can reduce blood viscosity, inhibit the production of oxygen free radicals and ameliorate and ischemia-reperfusion injury. Salvia miltiorrhiza can protect the intestinal mucosal barrier, improve macrophage activity and exhibits immunomodulatory function. Astragalus membranaceus has the effect of replenishing qi and strengthening the exterior, diuretics and detoxification, and can significantly improve the activity of superoxide dismutase in the plasma and small intestine, thereby reducing intestinal mucosal injury and endotoxin damage to organs ${ }^{[10-11]}$. The combination of various drugs together plays the role of clearing heat and detoxification, purging the Fu organs, promoting blood circulation, and removing blood stasis.

Our previous study showed that the administration of Tongfu Huoxue decoction to ICU patients with sepsis resulted in significant downregulation of plasma TNF-a, brain natriuretic peptide (BNP), plasma procalcitonin (PCT), C-reactive protein (CRP), and IL-10. Tongfu Huoxue decoction also protects the gastrointestinal mucosal barrier, promote blood circulation, and improve gastrointestinal dysfunction ${ }^{[12]}$. In this study, we used LPS, which is a vital component of Gram-negative bacteria, to establish a rat model of AKI in sepsis. This type of model is widely used for research purposes and mimics the pathological and physiological processes of sepsis in patients, with organ disorders occurring within 6-12 h of successful modeling ${ }^{[13]}$. After treatment with Tongfu Huoxue decoction, we found that the damage to the renal tubular epithelial cells was significantly reduced in model rats. Furthermore, SCr and BUN levels were significantly decreased following treatment with Tongfu Huoxue decoction, with a greater effect observed in the high-dose group compared with that in the low-dose group. Numerous pathophysiological studies on AKI in sepsis have confirmed the key role of inflammatory dysregulation in this condition and shown that high levels of inflammatory cytokines IL- 6 and TNF- $\alpha$ in the circulation are associated with an increased risk of mortality ${ }^{[14]}$. In this study we found that the expression of IL-1 $\beta$, IL- 6 and TNF-a in kidney tissue was downregulated after the rats were treated with Tongfu Huoxue decoction.

Sepsis is not only associated with high levels of cell necrosis, but also autophagy and apoptosis. LC3, which is a marker protein of autophagosome membrane that exists as type I and type II forms, is used as a biomarker of autophagy. Studies have shown that knockout of the key autophagy genes $L C 3$ and beclin-1, also affects the expression of the inflammatory factors IL-1 $\beta$ and IL-18 in mouse models of sepsis ${ }^{[15-16]}$. These findings indicate that autophagy plays an important role in the pathogenesis and inflammatory response associated with sepsis. Caspase-3 functions as an effector protease in apoptotic cell death by participating in the formation of apoptotic bodies that mediate apoptosis and regulate inflammatory pathways. The phosphoinositide 3-kinase (PI3K)/serine threonine protein kinase (Akt) signaling pathway regulates inflammatory responses mainly by modulating the expression of inflammatory mediators through Akt downstream proteins ${ }^{[17]}$, suggesting that Akt is a key protein in $\mathrm{PI} 3 \mathrm{~K}$ 
signaling pathways ${ }^{[18]}$. In this study we found that Tongfu Huoxue decoction significantly reduced the expression of LC3, beclin-1, caspase-3 and p-Akt in AKI model rat kidney tissues. The results of in vitro cell experiments also showed that Furthermore, in vitro studies showed that Tongfu Huoxue decoction reduced the levels of LC3 and Akt phosphorylation levels and reduced apoptosis in LPS-stimulated HK-2 cells.

\section{Conclusion}

our study showed that Tongfu Huoxue decoction reduced the degree of acute renal function injury in sepsis by downregulating the autophagy of renal tubular epithelium and reducing apoptosis of renal tubular epithelial cells. Thus, our findings provide a better understanding of the mechanism underlying the protective effect of Tongfu Huoxue decoction against AKI in sepsis and also provide a new strategy for early clinical intervention.

\section{Abbreviations}

PCR: Polymerase chain reaction

AKI: Acute kidney injury

CKD: chronic kidney disease

LPS: lipopolysaccharide

BUN: blood urea nitrogen

SCr. serum creatinine

HE: hematoxylin and eosin

BNP: brain natriuretic peptide

PCT: plasma procalcitonin

CPR: C-reactive protein

PI3K: phosphoinositide 3-kinase

Akt: serine threonine protein kinase

\section{Declarations}

\section{Ethics approval and consent to participate}


The experimental procedures were performed in accordance with the Principles of Laboratory Animal Care (NIH publication no.85Y23, revised 1996)and approved by the Animal Care Committee of Hebei Medical University.

\section{Consent for publication}

Written informed consent for publication was obtained from all participants.

\section{Availability of data and materials}

The datasets used and/or analyzed during the current study are available from the corresponding author on reasonable request.

\section{Competing interests}

All authors declared no competing interests.

\section{Funding}

Not applicable.

\section{Authors'Contributions}

YW and LD conceived and designed the study,YW and LD wrote the main manuscript text. ZW, QY, YF, and LW performed the animal experiments and collected data. ZW and $Y Z$ analyzed the data.LD prepared the figures and tables. All authors read and approved the final manuscript.

\section{Acknowledgements}

Not applicable.

\section{References}

[1] Harrois A, Grillot N, Figueiredo S, Duranteau J. Acute kidney injury is associated with a decrease in cortical renal perfusion during septic shock. Crit Care. 2018;22:161.

[2]Ma S, Evans RG, Iguchi N, Tare M, Parkington HC, Bellomo R, May CN, Lankadeva YR. Sepsis-induced acute kidney injury: A disease of the microcirculation.

Microcirculation. 2019; 26:e12483.

[3] Nisula S, Kaukonen KM, Vaara ST, Korhonen AM, Poukkanen M, Karlsson S, Haapio M, Inkinen O, Parviainen I, Suojaranta-Ylinen R, Laurila JJ, Tenhunen J, Reinikainen M, et al, and FINNAKI Study Group.Incidence, risk factors and 90-day mortality ofpatients with acute kidney injury in Finnish intensivecare units: the FINNAKI study. Intensive Care Med.2013; 39:420-28. 
[4] Schrier RW, Wang W. Acute renal failure and sepsis. NEngl J Med. 2004; 351:159-69.

[5] Nair AB, Jacob S. A simple practice guide for dose conversion between animals and human. J Basic Clin Pharm. 2016 Mar;7(2):27-31. doi: 10.4103/0976-0105.177703. PMID: 27057123; PMCID: PMC4804402.

[6]Kao CC, Yang JY, Chen L, et al. Factors associated with poor outcomes of continuous renal replacement therapy. PLoS One. 2017;12:e0177759.

[7] Fuchs L, Lee J, Novack V, et al. Severity of acute kidney injury and two-year outcomes in critically ill patients. Chest. 2013;144:866-875.

[8] Choi SJ, Ha EJ, Jhang WK, et al. Factors associated with mortality in continuous renal replacement therapy for pediatric patients with acute kidney injury. PediatrCrit Care Med. 2017;18:e56-e61.

[9]Alobaidi R, Basu RK, Goldstein SL, et al. Sepsis-associated acute kidney injury.SeminNephrol. 2015;35: 2-11.

[10]Huang T, Zhong LLD, Lin CY, Zhao L, Ning ZW, Hu DD, Zhang M, Tian K, Cheng CW, Bian ZX; for MZRW Research Group. Approaches in studying the pharmacology of Chinese Medicine formulas: bottom-up, top-down-and meeting in the middle. Chin Med. 2018 Mar 21;13:15. doi: 10.1186/s13020-018-0170-4. PMID: 29588653; PMCID: PMC5863461.

[11]Zheng Y, Ren W, Zhang L, Zhang Y, Liu D, Liu Y. A Review of the Pharmacological Action of Astragalus Polysaccharide. Front Pharmacol. 2020 Mar 24;11:349. doi: 10.3389/fphar.2020.00349. PMID: 32265719; PMCID: PMC7105737.

[12] Du LJ, Yin Z, Cui Y, Zhao R, Zhang W. A clinical study on Tongfu Huoxue Decoction in the treatment of sepsis gastrointestinal dysfunction. Hebei J TCM, 2012, (34), 7: 984-986.

[13] Park JT, Lee H, Kee YK, et al. High-dose versus conventional-dose continuous venovenous hemofiltration and patient and kidney survival and cytokine removal in sepsis-associated acute kidney injury: a randomized controlled trial. Am J Kidney Dis.2016;68:599-608.

[14]Alobaidi R, Basu RK, Goldstein SL, et al. Sepsis-associated acute kidney injury.SeminNephrol. 2015;35:2-11.

[15] Zamoner W, de Freitas FM, Garms DSS, et al. Pharmacokinetics and pharmacodynamics of antibiotics in critically ill acute kidney injury patients.Pharmacol Res Perspect. 2016;4:e00280.

[16]Doi K, Rabb H. Impact of acute kidney injury on distant organ function: recent findings and potential therapeutic targets. Kidney Int. 2016;89:555-564. 
[17]Ladda MA, Goralski KB. The effects of CKD on cytochrome P450-mediated drug metabolism.Adv Chronic Kidney Dis. 2016;23:67-75.

[18]Romagnoli S, Ricci Z, Ronco C. CRRT for sepsis-induced acute kidney injury.CurrOpinCrit Care. 2018; 24:483-92.

\section{Tables}

Table 1. Primer sequences

\begin{tabular}{ll}
\hline Gene & Primer sequence 5'-3' \\
\hline IL-1ן & Forward: GTACCTGAGCTCGCCAGTG \\
& Reverse: TGTTTAGGGCCATCAGCTT \\
IL-6 & Forward: CTACCAAGAACTGGCAATATG \\
& Reverse: AAAOCATCTGGCTAGGTAAGA \\
TNF- $\boldsymbol{\alpha}$ & Forward: GAGAGGCCGAGATTCACAGAG \\
& Reverse: CGGAACAAATCCAGAAGACCAG \\
$\boldsymbol{\beta}$ actin & Forward: GCCATGTACGTAGOCATCCA \\
& Reverse: GAAOCGCTCATTGOCGATAG \\
\hline
\end{tabular}

Table 2. Reverse transcription reaction system composition. Reaction conditions: $37^{\circ} \mathrm{C}$ for $60 \mathrm{~min}$ followed by $95^{\circ} \mathrm{C}$ for $5 \mathrm{~min}$ to inactivate the reverse transcriptase. The cDNA was stored at $-20^{\circ} \mathrm{C}$ for later use. 


\begin{tabular}{ll}
\hline Constituents & Content \\
\hline $5 \times$ RT Reaction Buffer & $5.0 \mu \mathrm{l}$ \\
Random nonamer primer $(0.2 \mu \mathrm{g} / \mu \mathrm{l})$ & $1.0 \mu \mathrm{l}$ \\
$\mathrm{M}-\mathrm{MLV}(200 \mathrm{U} / \mu \mathrm{l})$ & $0.5 \mu \mathrm{l}$ \\
RNase inhibitor & $1.0 \mu \mathrm{l}$ \\
$10 \mathrm{mmol} / \mathrm{L}$ dNTP & $0.5 \mu \mathrm{l}$ \\
Total RNA & $0.1-5 \mu \mathrm{g}$ \\
Add DEPC water to & $25 \mu \mathrm{l}$ \\
\hline
\end{tabular}

Table 3 Real-time PCR reaction system

Reaction conditions: $95^{\circ} \mathrm{C}$ for 3 min pre-denaturation, followed by 10 cycles of amplification at $95^{\circ} \mathrm{C}$ for $45 \mathrm{~s}$, and $55^{\circ} \mathrm{C}$ for $1 \mathrm{~m}$, and 30 cycles of amplification at $95^{\circ} \mathrm{C}$ for $30 \mathrm{~s}$, and $55^{\circ} \mathrm{C}$ for $45 \mathrm{~s}$.

\begin{tabular}{ll}
\hline Constituents & Volume $(\mu \mathrm{l})$ \\
\hline $10 \times$ PCR KCl Buffer & 5 \\
dNTP $(10 \mathrm{mmol} / \mathrm{L})$ & 1 \\
Taq DNA polymerase $(1 \mathrm{U} / \mu \mathrm{l})$ & 3 \\
$\operatorname{Mgcl}_{2}(50 \mathrm{mmol} / \mathrm{L})$ & 3 \\
Forward primer $(10 \mu \mathrm{mol} / \mathrm{L})$ & 1 \\
Rorward primer $(10 \mu \mathrm{mol} / \mathrm{L})$ & 1 \\
TaqMan Probe $(10 \mu \mathrm{mol} / \mathrm{L})$ & 0.5 \\
Positive Template cDNA & 2 \\
$50 \times$ ROX & 1 \\
Add dd $\mathrm{H}_{2} \mathrm{O}$ to & 50 \\
\hline
\end{tabular}

Figures 
A

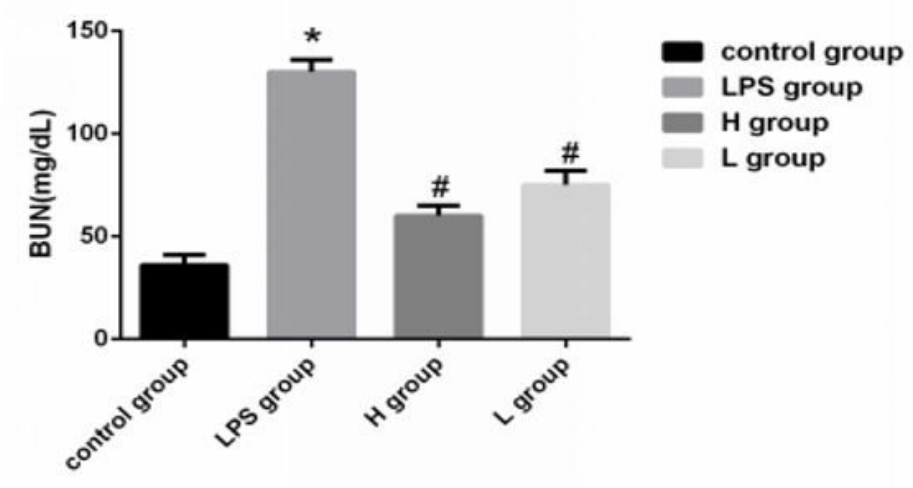

B

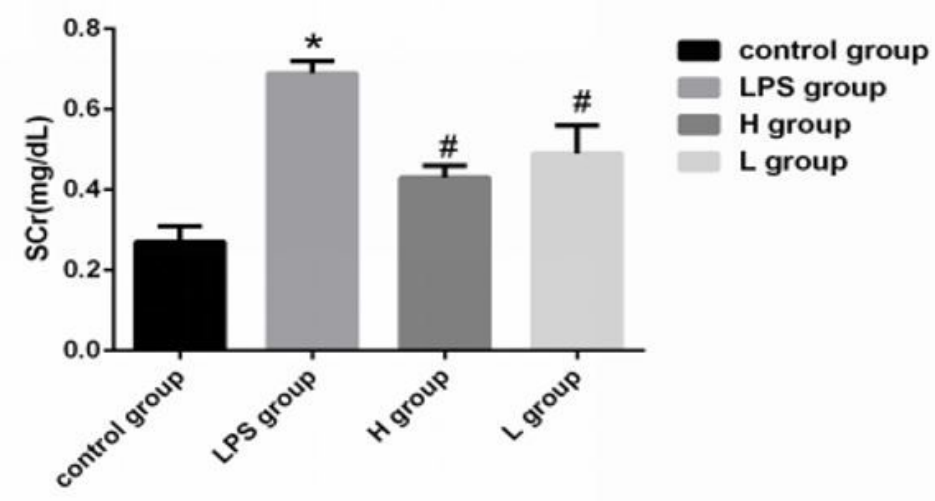

\section{Figure 1}

Blood urea nitrogen (BUN) and serum creatinine ( $\mathrm{SCr}$ ) levels in the rats in the control, LPS, LPS $+\mathrm{H}$ and LPS $+L$ groups. A: BUN; B: SCr冈Data represent the mean $\pm S D(n=3)$. ${ }^{*}<0.05$ vs. control group; $\# P<$ 0.05 vs. LPS group.

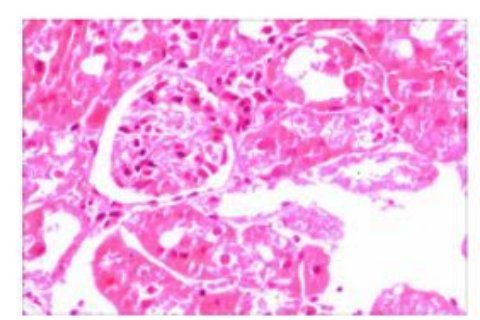

control group

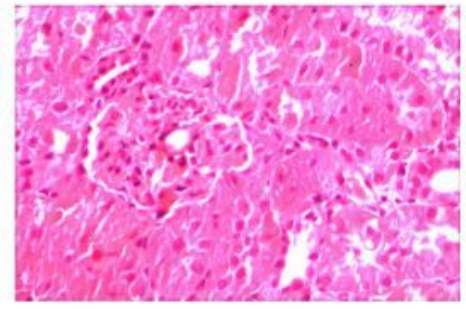

LPS group

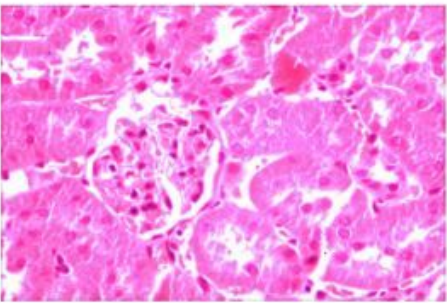

$\mathrm{H}$ group

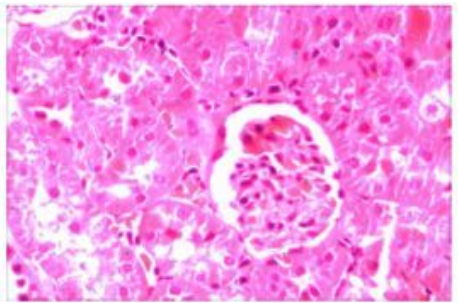

L group

\section{Figure 2}

The pathological changes of renal tissue in rats (HE staining *200).
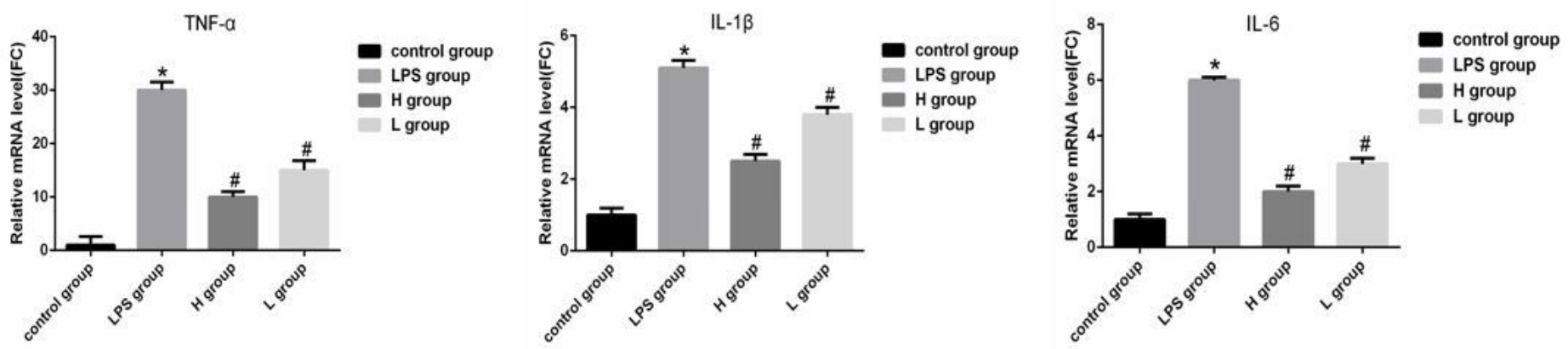

\section{Figure 3}

Quantitative real-time PCR analysis of proinflammatory cytokine gene expression in renal tissue. A: IL-1 3 .

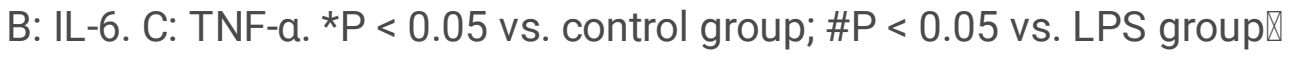



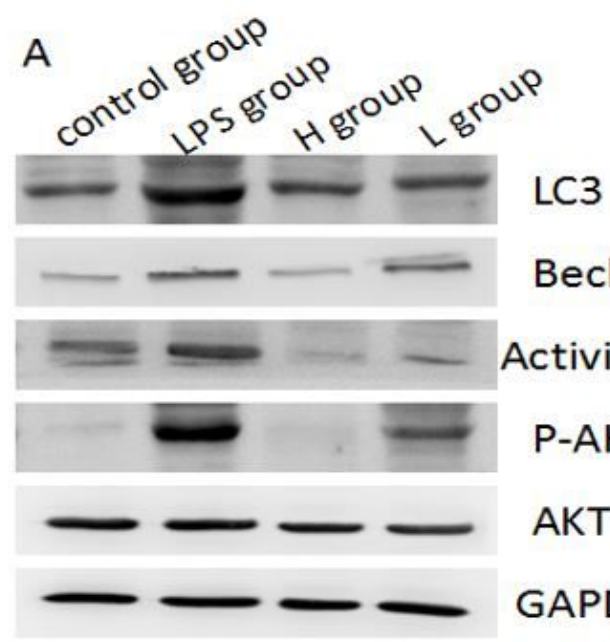

Beclin-1

Activivated caspase- 3

P-AKT

AKT

GAPDH
B

control group

LPS group

H group

L group

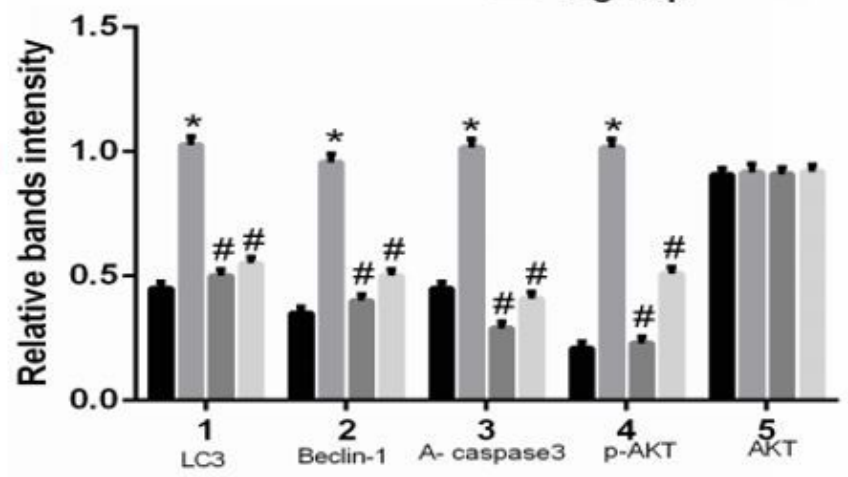

\section{Figure 4}

Western blot analysis of the expression of LC3, A-caspase3, beclin-1and, p-Akt proteins in the kidney tissue of the LPS group rats $\triangle A$ : Western blot showing detection of LC3, beclin-1, activated caspase-3, $p$ Akt, and Akt proteins. B: Densitometric quantification of the immunoreactive bands shown in A. Data represent the mean $\pm S D(n=3)$. ${ }^{*}<0.05$ vs. control group; \# $P<0.05$ vs. LPS group.

A

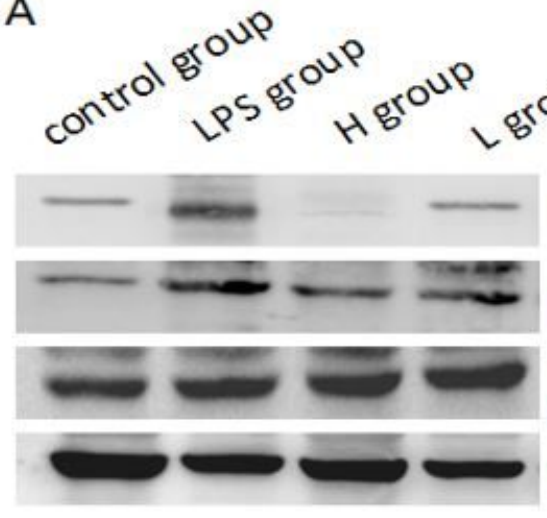

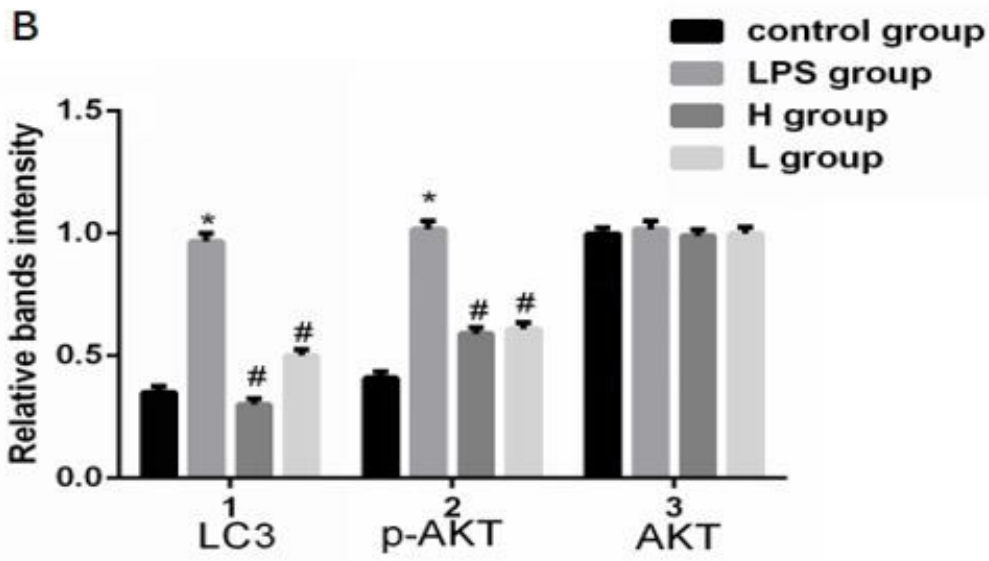

\section{Figure 5}

Western blot analysis of the expression of LC3 and p-Akt in HK-2 cells after LPS treatment. A: Western blot showing detection of LC3, p-Akt, and Akt in HK-2 cells after LPS treatment. B: Densitometric quantification of the immunoreactive bands shown in $A$. Data represent the mean $\pm S D(n=3) .{ }^{*}<0.05$ vs. control group; $\# \mathrm{P}<0.05$ vs. LPS group. 


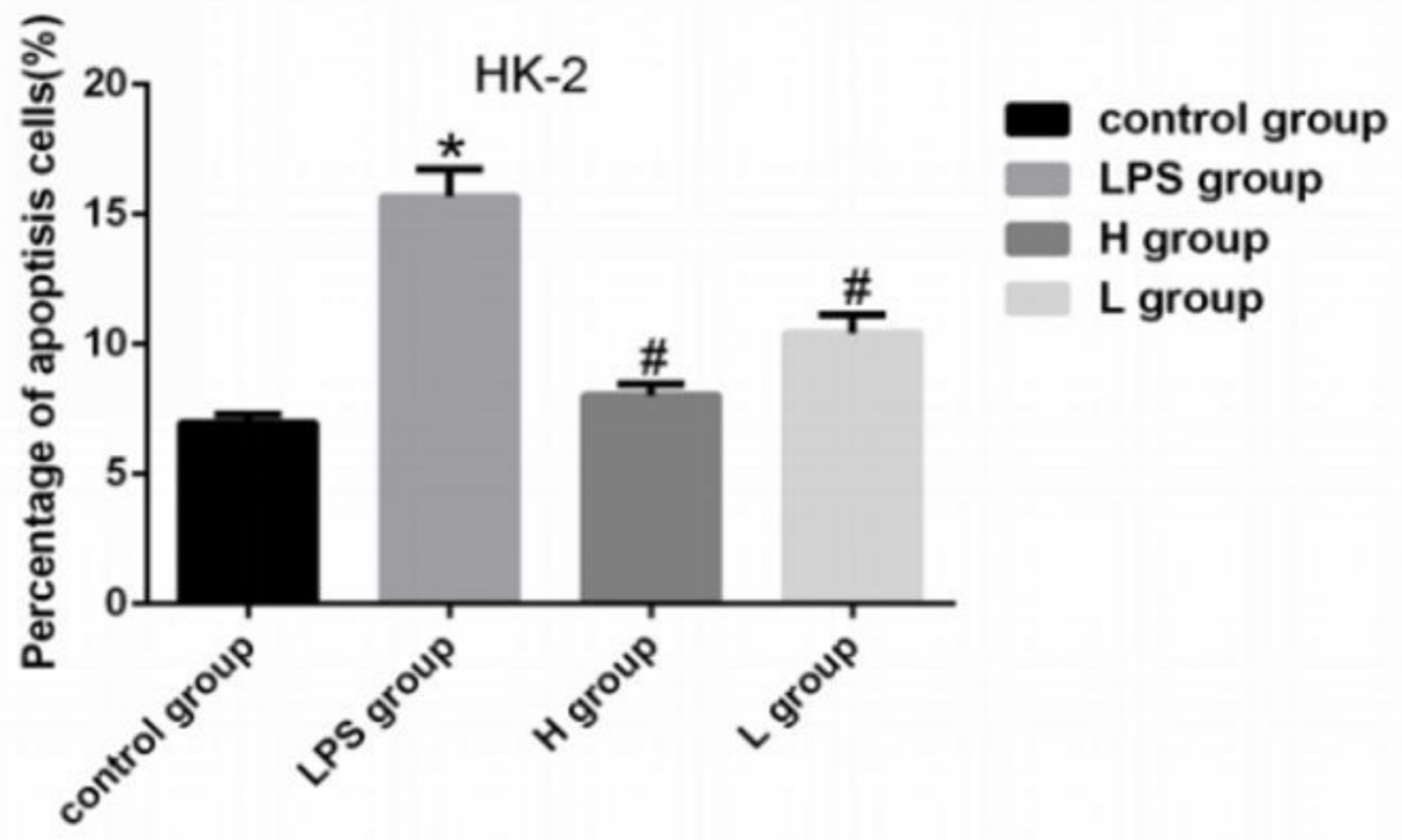

Figure 6

The effect of Tongfu Huoxue decoction on HK-2 cell apoptosis stimulated by LPS. Data represent the mean $\pm S D(n=3)$. ${ }^{*} P<0.05$ vs. control group; \# $P<0.05$ vs. LPS group. 